Corrigendum

\title{
Corrigendum to "The Prognostic Significance of Tumor Deposit Count for Colorectal Cancer Patients after Radical Surgery"
}

\author{
Kuo Zheng ${ }^{(D)},{ }^{1}$ Nanxin Zheng, ${ }^{1}$ Cheng Xin, ${ }^{1}$ Leqi Zhou, ${ }^{1}$ Ge Sun, ${ }^{1}$ Rongbo Wen, \\ Hang Zhang, ${ }^{1}$ Guanyu Yu, ${ }^{1}$ Chenguang Bai $\odot{ }^{2},{ }^{2}$ and Wei Zhang ${ }^{1}$ \\ ${ }^{1}$ Department of Colorectal Surgery, Changhai Hospital, Naval Medical University, Shanghai, China \\ ${ }^{2}$ Department of Pathology, Changhai Hospital, Shanghai, China \\ Correspondence should be addressed to Chenguang Bai; bcg709@126.com and Wei Zhang; weizhang2000cn@163.com \\ Received 28 July 2020; Accepted 28 July 2020; Published 19 September 2020 \\ Copyright (c) 2020 Kuo Zheng et al. This is an open access article distributed under the Creative Commons Attribution License, \\ which permits unrestricted use, distribution, and reproduction in any medium, provided the original work is properly cited.
}

In the article titled "The Prognostic Significance of Tumor Deposit Count for Colorectal Cancer Patients after Radical Surgery" [1], the authors "Kuo Zheng, Nanxin Zheng, Cheng Xin, Leqi Zhou, Ge Sun, Rongbo Wen, Hang Zhang, Guanyu $\mathrm{Yu}$, and Wei Zhang" were affiliated to the Department of Colorectal Surgery, Changhai Hospital, Shanghai, China which is incorrect. The correct affiliation for the authors is Department of Colorectal Surgery, Changhai Hospital, Naval Medical University, Shanghai, China. The corrected list of affiliations is shown above.

\section{References}

[1] K. Zheng, N. Zheng, C. Xin et al., "The prognostic significance of tumor deposit count for colorectal cancer patients after radical surgery," Gastroenterology Research and Practice, vol. 2020, Article ID 2052561, 8 pages, 2020. 